\title{
ABSCHIED VON DER PARLAMENTSBIBLIOTHEK UND VOM PARLAMENTSARCHIV: ELISABETH DIETRICH-SCHULZ TRAT MIT 1. SEPTEMBER 2020 IN DEN RUHESTAND
}

\author{
von Elisabeth Dietrich-Schulz und Oliver Schönsleben
}

Zusammenfassung: Elisabeth Dietrich-Schulz trat nach mehr als 40 Jahren im Bundesdienst, davon mehr als 30 Jahren Dienst in der Parlamentsdirektion ihren Ruhestand an. In dem vorliegenden Interview erzählt die langjährige Direktorin der Parlamentsbibliothek über den Wandel von Frauenkarrieren im Hohen Haus, ihren Lieblingsort im Parlament und ihre Ausdauer, mit der sie so manches Ziel erreichen konnte.

Schlagwörter: Elisabeth Dietrich-Schulz; Parlamentsbibliothek; Parlamentsarchiv; Interview

\section{FAREWELL TO THE AUSTRIAN PARLIAMENTARY LIBRARY AND ARCHIVES: ELISABETH DIETRICH-SCHULZ RETIRED ON SEPTEMBER 1, 2020}

Abstract: After more than 40 years in the civil service, including more than 30 years in the Austrian Parliamentary Administration Elisabeth Dietrich-Schulz retired as director of the parliamentary library and archives. In the following interview she talks about changes in the careers of women, her favorite place in the parliament building and the energy which enabled her to achieve many goals.

Keywords: Elisabeth Dietrich-Schulz; Austrian Parliamentary Library and Archives; Interview

DOI: https://doi.org/10.31263/voebm.v73i3-4.4329

(c) Elisabeth Dietrich-Schulz, Oliver Schönsleben

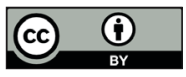

Dieses Werk ist - exkl. einzelner Logos und Abbildungen - lizenziert unter einer Creative-Commons-Lizenz Namensnennung 4.0 International-Lizenz 


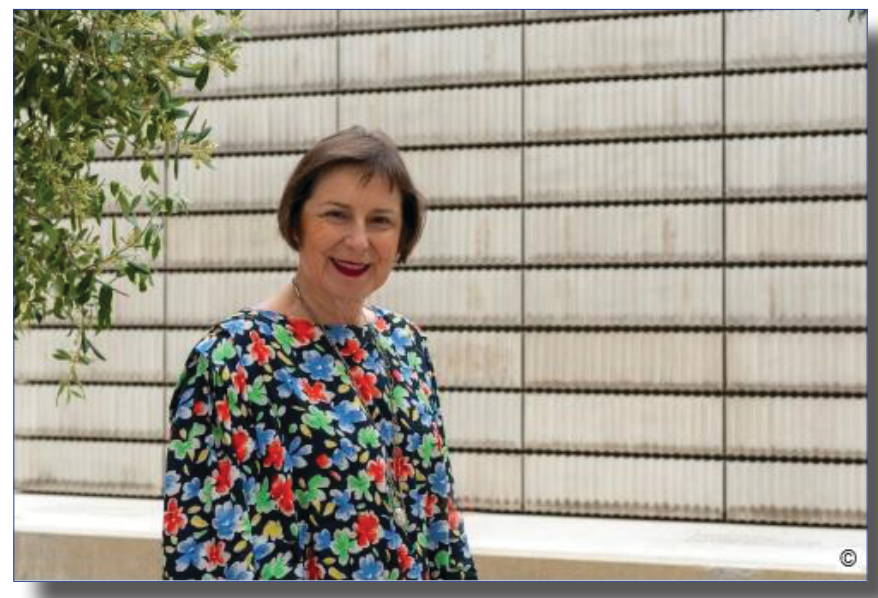

Abb.: Elisabeth Dietrich-Schulz (Foto: (c) Parlamentsdirektion / Michael Buchner, 2020)

Oliver Schönsleben: Sie haben 1989 Ihren Dienst in der Parlamentsdirektion angetreten. Auf welche persönlichen Highlights blicken Sie gerne zurück?

Elisabeth Dietrich-Schulz: Als ich im November 1989 meinen Dienst in der Parlamentsdirektion angetreten habe, habe ich mich sofort in den Lesesaal mit seinen hohen Eichenregalen und den Hansen-Tischen verliebt. Nur die vielen Metallkarteischränke haben mich gestört. Viel Arbeitszeit wurde damals in die Erstellung und Einordnung der Karteizettel investiert. Mein Ehrgeiz war, das zu ändern! Nachdem ich am 1. Jänner 1992 als erste Frau die Leitung der Parlamentsbibliothek übernehmen durfte, wurde noch 1992 der erste Computer angeschafft, ein Riesending! 1995 folgte der Umstieg auf ein EDV-gestütztes Bibliothekssystem. Seit 2001 sind alle Zettelkataloge der Bibliothek Vergangenheit und die Bestände in einer Datenbank abrufbar. 2003 wurde die Parlamentsbibliothek erste Kooperationspartnerin der Österreichischen Nationalbibliothek bei den wichtigen Digitalisierungsprojekten ANNO und ALEX. Historische österreichische Zeitungen und Zeitschriften sowie historische Rechts- und Gesetzestexte inklusive der Parlamentarischen Materialien ab 1848 wurden weltweit verfügbar, nicht zuletzt durch Beiträge der Parlamentsbibliothek. Seit 2012 ist die Bibliothek Mitglied des österreichischen Bibliothekenverbundes. Damit ist unser Bestand von über 370.000 Bänden und 43.000 Aufsätzen für die wissenschaftliche Forschung noch leichter zugänglich.

Persönliche Highlights waren Restitutionen im Rahmen der Provenienzforschung. Die Begegnung mit den aus Israel angereisten Erbinnen und 
Erben von Dr. Leopold Singer 2015 sowie der Besuch der Familie Blake 2019 waren besonders bewegend.

Meilensteine waren auch die erfolgreichen Verlagerungen von tausenden Büchern in die Rampenmagazine im Jahr 2006, die Übersiedlung von Bibliothek und Archiv ins Palais Epstein im Jahr 2017 und die Aufrechterhaltung des Bibliotheksbetriebes in Coronazeiten ab Mitte März 2020.

Das alles funktioniert nur mit einem tollen Team, dem ich an dieser Stelle aufrichtig danken möchte!

\section{Pionierin und Impulsgeberin}

Schönsleben: Sie waren zuvor im Bundesministerium für Landesverteidigung tätig. Welche Aufgaben hatten Sie dort inne?

Dietrich-Schulz: Auch im BMLV war ich eine Pionierin. 1978 wurde ich als erste Frau auf einen Akademikerinnenposten im Ministerium aufgenommen. Minister Otto Rösch hatte den Mut, das zu tun. Ich durfte an der Professionalisierung des Bibliotheksbetriebes und damit an der organisatorischen Aufwertung der Ministerialbibliothek und Vorschriftenverwaltung zur Präsidialabteilung D mitarbeiten. In diesen Jahren habe ich die Postgraduate-Ausbildung zur Bibliothekarin an der Österreichischen Nationalbibliothek und später den Führungskräftelehrgang Nr. 10 an der Verwaltungsakademie absolviert, wofür ich bis heute dankbar bin. Trotzdem hatte ich das Gefühl, im BMLV als Frau an die gläserne Karrieredecke zu stoßen. 1989 habe ich die große Chance bekommen, in die Parlamentsdirektion zu wechseln und hier neue Aufstiegschancen gefunden.

Schönsleben: Wie hat sich die Rolle der Frau seit Ihrem Dienstantritt geändert?

Dietrich-Schulz: Frauen wie Dr. ${ }^{\text {in }}$ Hilda Rothe oder Dr. ${ }^{\text {in }}$ Claudia Helbok wurden in der Parlamentsbibliothek bis in die 60er-Jahre des 20. Jahrhunderts trotz ihrer akademischen Ausbildung A-Posten und damit die finanzielle Anerkennung ihrer Leistungen verwehrt. Dr. in Brigitte Böck war von 1973 bis 1980 die erste Frau auf einem Akademikerinnenposten in der Bibliothek. 1992 wurde ich als erste Frau mit der Bibliotheksleitung und der stellvertretenden Leitung des parlamentarisch-wissenschaftlichen Dienstes betraut. Heute sind Frauen im Direktorium und als Dienstleiterinnen glücklicherweise selbstverständlich. Vieles konnte ich umsetzen und dabei auf der Arbeit meiner Vorgänger aufbauen. Im Buch „Nur was sich ändert, bleibt!: Die 
österreichische Parlamentsbibliothek im Wandel der Zeit 1869 - 2002“ ist vieles davon nachzulesen.

Schönsleben: Worauf sind Sie in Ihrer Tätigkeit in der Parlamentsdirektion besonders stolz?

Dietrich-Schulz: Mir ist es über weite Strecken gelungen, meine Begeisterung für das Buch und meinen Beruf zu leben und andere Menschen ebenso zu begeistern bzw. Impulse zu geben. Tatsächlich bin ich auf sehr viele Dinge stolz, z.B. auf die gelungene Erweiterung des Portfolios der Parlamentsbibliothek um die Aufsatzdokumentation und das Archiv. Die Bibliothek ist nicht nur die größte politische Fachbibliothek Österreichs, sondern dokumentiert die Publikationstätigkeit der Volksvertreterlnnen auf eine ganz besondere Weise. Veröffentlichungen von und über politische Mandatarinnen werden gesondert erfasst.

Wirklich stolz bin ich auf das Buch „Mark Twain. Reportagen aus dem Reichsrat 1898/1899“, den Bestseller des Hauses. Zwei Texte des amerikanischen Autors zum österreichischen Parlamentarismus circa 100 Jahre nach ihrem Entstehen zu finden und für ihre Übersetzung ins Deutsche zu sorgen, passiert einer Bibliothekarin, Anglistin und Historikerin nicht oft. Mit Hilfe zahlreicher Kolleginnen und Kollegen ist aus diesem Schatz 2017 ein Buch entstanden, das mit einem Vorwort von NR-Präsidentin Doris Bures im Residenzverlag erschienen ist und bereits ein Jahr später mit einem Vorwort von Nationalratspräsident Wolfgang Sobotka eine zweite Auflage erlebt hat.

Die europäische Vernetzung der Bibliothek war mir immer ein besonderes Anliegen. Als österreichische Korrespondentin des Europäischen Zentrums für Parlamentarische Wissenschaft und Dokumentation (EZPWD) habe ich bis 2020 fast täglich mit Kolleginnen und Kollegen von Aserbaidschan bis Zypern im Web zusammengearbeitet, über 3.000 Anfragen inhaltlich erledigt, zahlreiche Seminare organisiert und Vorträge gehalten. Bei der Zusammenarbeit der nationalen Parlamente Europas einen österreichischen Beitrag geleistet zu haben, erfüllt mich mit großer Freude. Die Tätigkeit war fordernd, aber spannend. Viele Kolleginnen und Kollegen sind Freunde geworden.

Ein weiterer Höhepunkt meiner Berufslaufbahn war die Einladung des US State Departments zur Teilnahme an einem dreiwöchigen Leadership Programm im Jahr 2009. Als erste österreichische Bibliothekarin durfte ich gemeinsam mit 21 Fachkolleginnen und Fachkollegen aus fünf Kontinenten US-Bibliotheken studieren. Im gleichen Jahr wurde ich von der UNO als 
Trainerin für ein einwöchiges Ausbildungsseminar für afrikanische Bibliothekarlnnen in Rom engagiert.

\section{Eindrucksvolles Parlamentsgebäude}

Schönsleben: Haben Sie einen Lieblingsort im Parlament?

Dietrich-Schulz: Natürlich! Die Säulenhalle! Oft dauerte der Dienst bei Plenarsitzungen bis Mitternacht oder länger. Dann bin ich am Heimweg gerne durch die halbdunkle Säulenhalle gegangen. Für Augenblicke hat der majestätische Raum mit seiner Mystik nur mir gehört!

Sehr beeindruckt haben mich die Feierlichkeiten anlässlich der Gedenktage gegen Gewalt und Rassismus. Seit Beginn 1998 bis 2019 habe ich sie alle miterleben dürfen. Die Aufführung der Oper „Das Tagebuch der Anne Frank" von Grigori Fried mit Anat Efrati im historischen Sitzungssaal hat mich am meisten bewegt.

Beim ersten „Tag der offenen Tür“ am 26. Oktober 1991 war ich genauso dabei wie beim letzten „Tag der offenen Tür“ 2016 vor Schließung des historischen Parlamentsgebäudes wegen der Sanierung. Diesen habe ich als etwas ganz besonderes in Erinnerung. So wurde u.a. das Abgeordneten-Sprechzimmer komplett ausgeräumt und mit Teppichen ausgelegt. Man durfte sich auf den Boden legen und bei Klaviermusik, den Raum genießen - ein besonders eindrucksvolles Erlebnis.

\section{Schönsleben: Was wünschen Sie sich zukünftig für die Parlamentsbibliothek?}

Dietrich-Schulz: Die Bibliothek wird nach der Rückübersiedlung in das historische Gebäude mit einigen Besonderheiten aufwarten. Das Kaffeehaus im neuen Besucherzentrum wird Bibliothekscafé heißen und ein breites Zeitungsangebot bieten. Ein Teil der Hansen-Bibliotheksregale aus dem 19.Jahrhundert wird - ähnlich dem Literaturmuseum - mit musealen Objekten und der Technologie des 21. Jahrhunderts digital zum Leben erweckt. Bibliothek und Archiv werden so zu Begegnungszonen zwischen Forschung und Öffentlichkeit. Anlässlich der Feier zum 150 Jahr-Jubiläum der Bibliothek im Herbst 2019 hat die Bibliothek mit ihrer Festschrift „Zu Wort gemeldet ist ... DAS BUCH“ eindrucksvoll bewiesen, wie wir uns das Be- und Erleben des einmaligen Bestandes vorstellen. Autorinnen und Autoren aus Politik, Wissenschaft, Medien und Kunst schreiben oder sprechen über „ihr“ Buch oder Archivobjekt und damit über ihr Verständnis von Parlamentarismus und Demokratie. 
Ich wünsche meinem Team, dass es auch in neuen Strukturen einen wertschätzenden Platz und Entfaltungsmöglichkeiten findet, denn das Potential und die Herausforderungen sind unglaublich groß.

Schönsleben: Die Parlamentsbibliothek verfügt über rund 370.000 Bücher. Haben Sie ein Lieblingsbuch?

Dietrich-Schulz: Mein Lieblingsbuch ist immer jenes Buch, das ich gerade lese. Auf meinem Nachtkästchen liegt derzeit „Zentrum der Welt. Das Forum Romanum als Brennpunkt der römischen Geschichte" von Wolfgang Buchner.

Sehr geprägt hat mich „Sehr blaue Augen“ der Literaturnobelpreisträgerin Toni Morrison. Ein Mädchen mit dunkler Augen- und Hautfarbe träumt davon, mit blauen Augen von einer rassistischen Gesellschaft akzeptiert zu werden.

\section{Good bye and good luck!}

Schönsleben: Wofür werden Sie ihre Freizeit nützen? Welchen Hobbies können Sie nun mehr Zeit widmen?

Dietrich-Schulz: Die Pandemie bremst meine Reiselust. Daher erkunde ich derzeit Wien, Niederösterreich und die Steiermark per Rad. Ich spiele leidenschaftlich gerne Volleyball, derzeit Beachvolleyball im Sand und auf der Wiese. Für den Herbst hoffe ich auf die Öffnung von Burg, Oper und Musikverein. Altersmäßig finde ich mich in der Risikogruppe und halte Abstand. Zum Glück bleibt mir Lesen mit vielen „Abenteuern im Kopf“.

Nachdem ich mich nicht - wie gehofft - bei lieben Kolleginnen und Kollegen persönlich verabschieden konnte, bedanke ich mich auf diesem Weg für die vielen Rückmeldungen auf meine Abschiedsmail. Diese „Briefe“ waren ein wunderbares Geschenk zum Abschied.

Good bye and good luck!

\section{Dr. ${ }^{\text {in }}$ Elisabeth Dietrich-Schulz Parlament, Parlamentsbibliothek, Direktorin i.R. E-Mail: eds.wien@gmail.com}

Oliver Schönsleben

Parlament, Pressedienst \& Cross-Media-Redaktion E-Mail: oliver.schoensleben@parlament.gv.at 